\title{
Tsafon
}

Revue d'études juives du Nord

$75 \mid 2018$

Exil des langues - Langues d'exil

\section{Discours du maire de Thessalonique}

Mémoire de la déportation des Juifs

\section{Yiannis Boutaris}

Traducteur : Consulat général de France à Thessalonique

\section{(2) OpenEdition}

Journals

Édition électronique

URL : https://journals.openedition.org/tsafon/626

DOI : $10.4000 /$ tsafon. 626

ISSN : 2609-6420

\section{Éditeur}

Association Jean-Marie Delmaire

\section{Édition imprimée}

Date de publication : 1 juin 2018

Pagination : 129-146

ISSN : 1149-6630

\section{Référence électronique}

Yiannis Boutaris, «Discours du maire de Thessalonique », Tsafon [En ligne], 75 | 2018, mis en ligne le

06 mai 2019, consulté le 01 juillet 2021. URL : http://journals.openedition.org/tsafon/626 ; DOI :

https://doi.org/10.4000/tsafon.626

Tsafon. Revues d'études juives du Nord 


\title{
Édition
}

\section{Discours du maire de Thessalonique}

\author{
Mémoire de la déportation des Juifs
}

Yiannis Boutaris*

Discours prononcé à l'occasion de la cérémonie de pose de la première pierre du musée mémorial de l'Holocauste (Thessalonique le 31 janvier 2018), en présence du Président d'Israël Reuven Rivlin, du Premier ministre grec Alexis Tsipras.

Autour de l'été 1945, Bouena Sarfati est sortie de chez elle. Juive âgée de 30 ans, Salonicienne "de père en fille », Bouena venait de rentrer à Thessalonique après avoir pris le maquis, au début avec l'EDES, puis l'EAM, puis s'était échappée en Palestine. Son frère Éliaou, sa sœur Regina, sa grand-mère centenaire Myriam et ses tantes n'ont pas eu la même chance. Un jour de printemps 1943, elles ont vu pour la dernière fois la ville qu'elles appelaient la «Jérusalem des Balkans», dans un wagon du train qui les transportait vers Auschwitz.

Quelques heures après leur arrivée, elles ont été conduites au crématorium, avec des milliers d'autres Juifs. Leur vie, et avec elle la vie

\footnotetext{
* Maire de Thessalonique. Traduction du Consulat général de France à Thessalonique. Présentation par Danielle Delmaire. Le titre et le sous-titre sont de Tsafon.

«Mes plus vifs remerciements vont à Monsieur le maire de Thessalonique et à Monsieur le Consul général de France à Thessalonique pour m'avoir communiqué ce texte, ainsi que sa traduction, et pour m'avoir permis de le publier et de le présenter » (Danielle Delmaire, université de Lille SHS).
} 
de la Thessalonique juive, de notre Thessalonique, s'est transformée en cendres dispersées dans les plaines inhospitalières de Pologne.

Est-ce que les proches et la famille de Bouena étaient des martyrs ? Est-ce que nous les honorons en nous souvenant d'eux comme tels ? Sommes-nous honorés de nous souvenir d'eux de cette façon ?

Aujourd'hui, cette « journée dédiée à la mémoire des martyrs juifs de l'Holocauste » nous invite à réfléchir sur cette question. Les membres de la famille de Bouena, comme tous les autres Juifs d'Europe, n'ont pas choisi d'être des martyrs. Ils n'ont pas choisi de sacrifier consciemment leur vie pour une cause, leur foi religieuse ou leur idéologie. Ils n'ont pas choisi la mort, tout simplement car ils n'avaient pas le droit de choisir. Et pour cette raison, ils ne méritent pas qu'on les traite aujourd'hui de saints, nous tous, chrétiens et Européens, qui pendant des siècles les considérions comme des diaboliques. C'étaient des êtres humains et c'est ce qu'ils voulaient être.

Certains, peu nombreux, comme Bouena, ont survécu: seuls quelques milliers de Thessaloniciens, survivants d'une communauté de plus de 45000 âmes. Ils ont survécu à la déportation à Auschwitz, à la marche de la mort, aux camps de travail. Ils ont survécu car ils ont résisté à la violence inexprimable, aux humiliations, aux expérimentations médicales, aux viols. Apres avoir survécu, ils sont revenus dans leur ville natale. Comme des héros? Bien au contraire. Des Juifs qui s'étaient échappés dans les montagnes, s'étaient cachés dans les villes ou qui s'étaient évadés en Palestine considéraient tous ceux et celles qui étaient revenus des camps de concentration comme des traitres, des collaborateurs, les femmes comme des prostituées. De leur côté, les chrétiens considéraient les survivants comme des "savons inexploités » selon le rapport d'un journaliste américain, une menace d'un passé qui ne voulait pas mourir.

Selon le journal Elliniko Vora, seuls cinq jeunes étaient des héros juifs qui, après avoir combattu sur le front albanais et après avoir survécu aux crématoriums, sont tombés en octobre 1948 « héroïquement lors des batailles des montagnes, alors qu'ils combattaient les ennemis ».

Pour Bouena, le martyre et l'héroïsme avaient peu de valeur lorsqu'elle essayait de rassembler ses morceaux et de reconstruire sa vie depuis le début. Comment pouvait-elle se sentir quand même les plus petits plaisirs ouvraient les plaies du passé. Combien lourde devait être sa peine en ce dimanche après-midi, lorsqu'elle découvrait que ses pois chiches secs préférés, qu'elle avait achetés au vendeur arménien, étaient 
emballés dans un cône de papier fait d'une page de l'Ancien Testament de sa famille.

Ce papier déchiré est le passé de Bouena, mais aussi le passé de notre ville, un passé qui nous poursuit et nous hante. C'est un passé silencieux, invisible mais présent. C'est la cour de l'église saint Dimitri dont les dalles de marbre proviennent des pierres tombales du cimetière juif de la ville, cimetière détruit par des Allemands et des employés municipaux chrétiens, un matériel "sans valeur» selon l'archéologue Stylianos Pelekanidis. C'est l'hôpital AHEPA et l'université Aristote qui ont été construits sur une des nécropoles les plus importantes d'Europe. Ce sont les pierres tombales juives qui ont été utilisées pour le dallage devant le commandement militaire et autour du théâtre royal, celles qui ont été utilisées par la municipalité de Thessalonique en novembre 1948 pour construire des routes et des trottoirs, malgré les intenses protestations de la communauté juive. Ce sont les pierres tombales entassées à la vue de tous devant la Tour blanche et dans la cour de la foire internationale jusqu'en décembre 1948. C'est le sac argenté, relique de famille, que Bouena Sarfati remarque avec surprise en 1946, au bras d'une amie de famille chrétienne. C'est le tapis familial qu'une autre Juive rescapée voit avec surprise, dans la maison d'une famille chrétienne amie. C'est le livre qui a été retrouvé par hasard, il y a à peine une dizaine d'années, dans la bibliothèque de «l'association caritative des Thessaloniciens » avant d'être rendu au musée juif, acte qui honore cette association.

Qui sont ceux qui, en 1945, ont pleuré leurs voisins disparus? Quels monuments ont été érigés ? Quelles cérémonies ont eu lieu ? Seule, la communauté torturée et rackettée s'est battue pour se reconstruire et pour pleurer ses morts. La ville, la société, le pays tout entier ont fait preuve d'indifférence. Ils se sont tous cachés derrière leur petit doigt. Ils ont fait comme s'ils ne savaient pas ce qui s'était passé, qui l'avait fait, qui avait aidé, qui avait protégé quand d'autres, nombreux, détruisaient, brûlaient, volaient, prenaient possession de l'espace et des biens des nombreux absents et des rares présents. Le deuil est de toute façon une affaire personnelle. Il a fallu attendre près de vingt ans pour qu'en 1962 soit construit un monument en mémoire des victimes. Où? Dans l'enceinte du nouveau cimetière juif de la ville, comme si cela ne concernait que les familles et les membres de la communauté juive. Quand 35 ans plus tard, un vrai monument a été érigé sur la place publique, il a été placé à la périphérie du centre-ville, dans un lieu peu 
visible. Quand enfin, ce monument a été déplacé dans son environnement naturel, sur la place de la Liberté, cela a suscité plus de surprise que de satisfaction.

Il a fallu attendre 2004 pour que le parlement grec vote à l'unanimité la journée du souvenir. Il a fallu attendre 2011 pour qu'un jour du souvenir soit célébré à Thessalonique et 2014 pour qu'une stèle commémorative soit érigée dans l'enceinte de l'université Aristote afin de rappeler la destruction du cimetière. Et peut-être que le jour n'est pas loin où nous verrons une plaque commémorative dans la cour de l'église Saint-Dimitri «le Saint Dimitri des morts juifs » dans le vrai mausolée juif de Thessalonique.

La municipalité de Thessalonique a de plus en plus conscience du poids de l'histoire que la ville doit soulever. Maintenant que les survivants nous quittent et que le relais de la mémoire passe dans les mains de nous toutes et tous, la municipalité a l'intention de continuer de transformer le silence en mots, mots de consolation, mais aussi mots de courage. Nous voulons que le réaménagement de la place de la Liberté et le musée de l'Holocauste constituent le nouvel axe de mémoire de la ville, le point de départ et l'aboutissement du grand parcours multiculturel, chrétien, musulman, juif de Thessalonique. La place de la Liberté est un lieu de démocratie où, en 1908, tous les Thessaloniciens musulmans, chrétiens et juifs ont fêté, ensemble, la proclamation de la constitution ottomane. Il s'agit aussi d'un lieu de déracinement et d'exil, le lieu d'où sont partis en 1922-1923 les anciens Thessaloniciens musulmans et où ont débarqué les nouveaux réfugiés d'Asie Mineure et du Pont-Euxin. Mais c'est aussi un lieu de martyre, d'humiliation publique des Juifs thessaloniciens, où le samedi noir du 11 juillet 1942, les Allemands ont diffamé 9000 Juifs devant les chrétiens grecs.

Cette place représente un lieu gênant. Elle nous rappelle que l'Holocauste de Thessalonique est le plus lourd des maillons d'une longue chaîne de violence et d'humiliation. Elle nous rappelle que ses Juifs étaient un morceau indissociable d'une mosaïque colorée, que la «Jérusalem des Balkans» était en même temps la «Babel de la Méditerranée ».

Nous voulons que la place de la Liberté soit le lieu où les souvenirs difficiles, traumatisants de tous les habitants de cette ville ne se concurrencent pas mais au contraire cohabitent en harmonie: ils dialogueront vivement pour promouvoir une culture de cohabitation et de respect mutuel afin que le lourd héritage du passé se transforme en 
tremplin pour un futur meilleur. La nouvelle place de la Liberté va symboliser la fierté de tous les Thessaloniciens pour leur ville, son passé, son présent et son futur.

À quelques mètres de là, le musée de l'Holocauste symbolisera notre honte pour tout ce qui s'est passé, pour tout ce que nous avons fait et surtout pour tout ce que nous n'avons pas pu ou pas voulu faire, autochtones et réfugiés, de droite ou de gauche, pendant et après la guerre. Le musée répond à une dette de la ville mais constitue également un pari personnel. C'est une dette de la ville envers ses Juifs, en tant que Thessaloniciens, Grecs et Sépharades.

Le musée dépasse la ville et la Grèce et replace Thessalonique comme la métropole des Juifs sépharades de Méditerranée. Son ambition est de raconter l'histoire méconnue de l'Holocauste des Juifs de Méditerranée et des Balkans, des Juifs sépharades de Thessalonique et de Corfou, de La Canée et de Patras, mais aussi de Belgrade, de Skopje, de Monastir et de Sarajevo, de Trieste et de Libourne. Il aspire à transformer la page déchirée de Bouena en connaissance historique, à mettre en valeur un aspect de l'Holocauste qui est souvent ignoré à cause de son ampleur en Europe centrale et orientale. Et de cette façon, instituer Thessalonique en un lieu de mémoire mais aussi en un centre de recherche et d'étude d'envergure internationale. Et enfin, il aspire à devenir un lieu où les habitants de la terre entière, en particulier les jeunes, apprendront les conséquences du bafouement des droits de l'homme.

Nombreux sont ceux qui nous demandent pourquoi, pourquoi donner de l'importance à l'histoire et au souvenir des Juifs de Thessalonique ? La profanation du monument de l'Holocauste dimanche dernier et l'incendie d'un bâtiment historique ayant appartenu à une Juive thessalonicienne suffiraient comme réponse. Mais personnellement, je préfère répondre en reprenant une phrase de Primo Levi. «Ici, il n'y a pas de pourquoi » lui a répondu le garde allemand dès qu'il est arrivé à Auschwitz. Ici il n'y a pas de pourquoi, je pourrais répondre moi aussi à tous ceux qui s'étonnent de mon insistance. L'Holocauste des Juifs d'Europe, l'Holocauste de nos Juifs dépasse les limites de la logique et la seule façon de se mesurer à lui est d'accepter le fait qu'il fera toujours partie de ce que nous sommes en tant que Thessaloniciens, Grecs et Européens : une page déchirée écrite d'une écriture inconnue, une vérité qui attend encore d'être déchiffrée.

\footnotetext{
$* * * * * * * * * * * * *$
} 


\section{Présentation par Danielle Delmaire ${ }^{1}$}

Il y a six mois à peine, Yiannis Boutaris, maire de Thessalonique, prononçait un émouvant discours sur la déportation et l'extermination des Juifs de Thessalonique - qu'il appelle « Holocauste » comme le font les historiens anglo-saxons et non Shoah comme nous le faisons en France et comme le font les Israéliens - lors de la pose de la première pierre du musée national de l'Holocauste, très officiellement puisque la plus haute autorité représentant l'État d'Israël, son président Reuven Rivlin, et le Premier ministre grec, Alexis Tsipras, étaient présents. La municipalité de Thessalonique qui fut « la Thessalonique juive» se trouvait alors être le lien entre la Grèce et Israël.

Yiannis Boutaris, maire de Thessalonique, et le passé juif de la ville

Né en 1942 à Thessalonique, homme d'affaires et producteur de vins de la région, Yiannis Boutaris est entré tardivement en politique en briguant le poste de maire de la seconde ville de Grèce, en 2010. N'appartenant pas à un parti politique, il est l'homme du renouveau de la Grèce : «humaniste et anticonformiste», il symbolise «le vent du changement qui souffle sur la Grèce » n'hésite pas à écrire Jean-Paul Taillardas dans le journal Sud-Ouest, le 7 août $2013^{2}$. Élu de justesse sur un programme de "grand renversement », pour reprendre les mots cités par le même journaliste, il mit fin à un clientélisme coûteux pour la ville qui salariait des employés municipaux improductifs et il s'opposa fermement au métropolite orthodoxe Mgr Anthimos, conservateur et nationaliste extrémiste, ce qui lui assura le soutien de la gauche. Il est « le Grec qui donne l'exemple, celui qui porte l'espoir d'un renouveau [...] comme Hercule dans la mythologie, il nettoie les écuries d'Augias » ${ }^{3}$. Selon Die Zeit, il est « l'antithèse de l'homme politique » car lors de son élection à la mairie de Thessalonique il n'a pas un passé politique mais il est bien décidé à réformer et ouvrir sa ville à d'autres

\footnotetext{
${ }^{1}$ Université de Lille, SHS. Sauf indication contraire, les citations présentées entre guillemets proviennent du discours du maire.

${ }^{2}$ Article paru sur le site www.sudouest.fr.

${ }^{3}$ Ibid.
} 
activités, comme le tourisme ou le développement des productions agricoles locales, délaissées par ses prédécesseurs ${ }^{4}$.

Seconde ville de la Grèce, Thessalonique mérite bien son qualificatif de «Jérusalem des Balkans» pour l'importance de la présence juive, majoritaire dans la population à la fin du $\mathrm{XIX}^{\mathrm{e}}$ siècle et au début du XX ${ }^{\mathrm{e}}$. Depuis l'époque de Paul de Tarse, le juif Shaul, qui parla dans la synagogue de Thessalonique (Actes des apôtres 17,1) et qui écrivit deux épîtres aux Thessaloniciens, jusqu'à la veille de la Seconde Guerre mondiale les juifs ont eu en permanence un quartier avec écoles et synagogues, et ont exercé diverses activités économiques. À partir de la fin $\mathrm{du} \mathrm{XV}^{\mathrm{e}}$ siècle, la communauté juive prit encore plus d'importance avec l'arrivée des juifs sépharades expulsés de la péninsule ibérique : Thessalonique devint «la métropole des juifs sépharades de Méditerranée ». Le quartier juif de Thessalonique, en bordure du port donnant sur la mer Égée, s'enrichit de nombreuses synagogues, jusqu'à une soixantaine au début du $\mathrm{XX}^{\mathrm{e}}$ siècle $^{5}$; la culture judéo-espagnole s'y développa et la langue y est encore parlée jusqu'à nos jours. Les juifs participèrent activement à l'essor économique du port. $\mathrm{Au} \mathrm{XIX}^{\mathrm{e}}$ siècle, des familles s'adonnaient au commerce lointain, ouvraient des banques, implantaient des industries textiles, des tanneries etc. Cette croissance économique s'accompagna d'un développement culturel mettant en valeur le patrimoine judéo-espagnol apporté par les juifs de la péninsule ibérique. L'Alliance israélite universelle amplifia le rayonnement de la communauté juive en y implantant une école, à la fin du XIX ${ }^{\mathrm{e}}$ siècle, qui était fréquentée en 1908 par 1258 garçons et 825 filles $^{6}$. Les juifs faisaient entièrement partie de la population thessalonicienne, côtoyant d'autres communautés grecques chrétiennes, turques musulmanes et les dönmes (juifs convertis à l'islam après le passage dans la ville de

\footnotetext{
${ }^{4}$ Die Zeit, 8 mars 2012, paru sur le site www.voxeurop.eu. Le bénéfice de sa politique hardie fut reconnu par sa facile réélection en 2014. Sur Yiannis Boutaris, lire Pierre Sintès et Olivier Givre, «Iannis Boutaris et les fantômes de Salonique », Écrire l'histoire, ${ }^{\circ} 15$, Paris, éd. du CNRS, 2015, p. 215-222. Remerciements au Consul de France à Thessalonique pour m'avoir donné à lire cet article.

${ }^{5}$ Christos Zafiris, La Thessalonique juive, guide historique, Athènes, Epikentro, 2017, p. 102 (traduit du grec par Jonathan Zemour). Sur l'histoire de Thessalonique, se reporter à Esther Benbassa (dir.), Salonique. Ville juive, ville ottomane, ville grecque, Paris, éd. du CNRS, 2014 ; Gilles Veinstein (dir.), Salonique 1850-1918. La "ville des Juifs » et le réveil des Balkans, Paris, éd. Autrement, 1992.

6 Jacques Bigart, secrétaire général de l'Alliance israélite universelle, rapport cité dans le Dictionnaire Ferdinand Buisson. Voir le site électronique : www.inrp.fr/editionelectronique/lodel/dictionnaire-ferdinand-buisson/document.php?id=2020 Alliance israélite universelle écoles, consulté le 5 avril 2018.
} 
Shabbataï Zvi, prédicateur juif qui se présentait comme le Messie et finit par adhérer à l'islam, au XVII ${ }^{\mathrm{e}}$ siècle).

C'est la raison pour laquelle, Y. Boutaris peut les appeler «nos juifs » et affirmer « que ses juifs [de Thessalonique] étaient un morceau indissociable d'une mosaïque colorée, que la 'Jérusalem des Balkans' était en même temps la 'Babel de la Méditerranée'».

\section{La rupture meutrière de la Seconde Guerre mondiale}

Dès lors que les Allemands furent maîtres de Thessalonique, les humiliations et les persécutions envers les juifs commencèrent: les responsables communautaires furent arrêtés, des familles furent spoliées de leurs biens, de leurs demeures et de leurs établissements économiques, de leurs œuvres d'art, et les synagogues furent pillées. Les hommes âgés de 18 à 45 ans durent subir l'éprouvante humiliation du « shabbat noir » : le 11 juillet 1942, ils furent rassemblés sur la place de la Liberté, au centre de la ville, pour y effectuer des exercices physiques aussi inutiles qu'épuisants, sous le soleil de l'été méditerranéen. Puis, les Allemands affectèrent 2000 juifs à des travaux forcés de terrassement, ceux-ci furent libérés contre une rançon versée par la communauté juive. Enfin, avant sa déportation, la population juive fut enfermée dans deux ghettos, l'un recouvrant une importante superficie avec l'hôpital juif réquisitionné et l'autre plus petit, le ghetto Hirsch, dans le quartier près de la gare portant le nom du bienfaiteur des juifs pauvres ${ }^{7}$.

Du 15 mars au 10 août 1943, dix-neuf convois déportèrent 48533 juifs de Thessalonique ${ }^{8}$, parmi lesquels se trouvaient les membres de la famille de Bouena Sarfati qu'énumère Yiannis Boutaris : "Son frère Éliaou, sa sœur Regina, sa grand-mère centenaire Myriam et ses tantes [...]. Un jour de printemps 1943, elles ont vu pour la dernière fois la ville [...] dans un wagon du train qui les transportait vers Auschwitz » et il ajoute un peu plus loin : «C'étaient des êtres humains, et c'est ce qu'ils voulaient être ». 11079 déportés furent sélectionnés pour le travail, les autres furent directement dirigés vers les chambres à gaz ${ }^{9}$ puis « aux crématoriums $[\ldots]$ quelques heures après leur arrivée ». Cette déportation

\footnotetext{
${ }^{7}$ La déportation des juifs de Thessalonique selon un résumé établi par le Mémorial de la Shoah.

${ }^{8}$ Bilan des convois partis de Thessalonqiue vers Auschwitz établi par le Mémorial de la Shoah.

${ }^{9}$ Ibid.
} 
massive touchait la quasi-totalité de la population juive de Thessalonique: «...La vie de la Thessalonique juive, de notre Thessalonique, s'est transformée en cendres dispersées dans les plaines inhospitalières de Pologne $»$.

Très peu d'entre eux ont survécu : « ... à la marche de la mort, aux camps de travail [...] à la violence inexprimable, aux humiliations, aux expérimentations médicales, aux viols ». Lucidement, Yiannis Boutaris mesure la part de responsabilité de l'enseignement des Églises chrétiennes, ici principalement l'Église orthodoxe, dans cette catastrophe : «...Nous tous, chrétiens et Européens, qui pendant des siècles les considérions comme diaboliques ». Certes, c'est l'idéologie nazie qui incita les dirigeants allemands à mettre en pratique l'extermination des juifs mais cette idéologie prenait sa source, en grande partie, dans les accusations de déicide et de malfaisance portées contre les juifs par l'ensemble des chrétiens, depuis deux millénaires. Les souffrances des survivants ne les exonéraient pas d'une perpétuelle hostilité : "De leur côté, les chrétiens considéraient les survivants comme des 'savons inexploités' [...] une menace d'un passé qui ne voulait pas mourir ». Comme souvent, le maire de Thessalonique n'hésite pas à appuyer le propos pour interpeller ses concitoyens. Dans les camps d'extermination, les nazis ont récupéré tout ce qui était récupérable des corps de leurs victimes: les chevelures exploitées pour fabriquer des tissus, la peau transformée en matière pour fabriquer des abat-jour, aussi la graisse pour des savons! Les survivants d'Auschwitz n'avaient donc pas pu être recyclés en « savons ».

Quelques juifs comprirent rapidement la menace dévastatrice qui s'annonçait lorsque les Allemands devinrent les maîtres de la ville et parfois même avant. D'aucuns ont fui vers Athènes en zone contrôlée par les Italiens qui, comme en France, n'ont pas mené de politique antisémite et ont même aidé les juifs à trouver des refuges parmi eux ${ }^{10}$. Ce fut le cas du consul d'Italie à Thessalonique, Emilio Neri, qui organisa le transfert de plusieurs juifs vers la zone italienne, avec la complicité de cheminots qui acceptaient de les cacher dans leurs transports de marchandises ${ }^{11}$. Il

\footnotetext{
${ }^{10}$ Que l'on se souvienne, entre autres témoignages, du récit de Joseph Joffo qui évoque la bonne entente entre les Italiens et les juifs, à Nice, sous l'occupation italienne. Joseph Joffo, Un sac de billes, Paris, Hachette, 1983. Par la suite, le récit a été réédité de nombreuses fois et adapté dans des collections pour enfants et au cinéma.

${ }^{11}$ Martin Gilbert, Les Justes : les héros méconnus de la Shoah, Paris, Calmann-Levy, 2004, traduit de l'anglais par Élie-Robert-Nicoud.
} 
fournit même un passeport à Bouena Sarfati ${ }^{12}$ qui entra en résistance avec d'autres coreligionnaires.

\section{La résistante et la poétesse Bouena Sarfati (1916-1997)}

Elle revint donc à Thessalonique en 1945, sans sa famille dont les ancêtres étaient des Thessaloniciens depuis longtemps. Bouena Sarfati était «Salonicienne 'de père en fille'». Elle avait évité le massacre car elle avait «...pris le maquis, au début à l'EDES, puis à l'EAM, puis s'était échappée en Palestine ».

Dès 1940, l'Italie tenta de prendre pied en Grèce à partir de l'Albanie mais son armée se heurta à une résistance de la part de la Grèce qui intégra des soldats juifs dans son armée : «Cinq jeunes étaient des héros juifs [qui avaient] combattu sur le front albanais ». Néanmoins, dès avril 1941, les forces de l'Axe occupèrent la Grèce: les Italiens s'installèrent dans toute la péninsule tandis que les Allemands et les Bulgares se partagèrent le rivage sud des Balkans, Thessalonique tombait sous domination allemande. En juillet et septembre 1943, les Allemands maîtrisèrent toute la Grèce, évinçant ses alliés.

Dès le début de la présence allemande dans le nord, à l'automne 1941, la résistance à l'occupant s'organisa. Elle se partagea entre plusieurs mouvements dont l'EDES (Ligue nationale démocratique grecque) de tendance républicaine et l'EAM, émanation du parti communiste. Chacun de ces deux mouvements créa, en 1942, une branche armée et développa des maquis dans toute la Grèce.

L'itinéraire de Bouena fut donc celui d'autres Grecs, non juifs et juifs. Elle avait « ...pris le maquis, au début dans l'EDES, puis l'EAM » alors que les deux mouvements n'avaient pas encore conclu un accord, décidé en mai 1943, pour mener des attaques communes. Ensuite, sans que Yiannis Boutaris en donne la raison, elle «...s'était échappée en Palestine », encore placée, à cette époque, sous le mandat britannique. Le voyage dut s'effectuer clandestinement puisqu'elle bénéficia d'une aide du consul d'Italie et que les Anglais combattaient l'Axe sur le front sud de la Palestine, vers l'Égypte. Son épopée ne fut pas unique : « Des Juifs qui s'étaient échappés dans les montagnes, s'étaient cachés dans les villes ou qui s'étaient évadés en Palestine...». Les maquis, facilités par la nature montagneuse de la Grèce, accueillirent des juifs, peu nombreux car à partir de 1942 il leur fut peu aisé de sortir des ghettos. De même, il

\footnotetext{
${ }^{12}$ Ibid.
} 
était très difficile pour les juifs de trouver de l'aide dans la population non juive sans doute à cause de l'antisémitisme latent mais aussi du fait qu'ils étaient trop nombreux pour vivre clandestinement dans une ville quadrillée par l'occupant.

Bouena Sarfati n'effectua pas son alyah en Palestine puisqu'elle revint à Thessalonique dès 1945, mais ce fut pour constater que « la Thessalonique juive [...] s'est transformée en cendres...». Elle ne resta pas sur ces « cendres », par la suite elle émigra au Canada, à Montréal, où elle mit en valeur son patrimoine culturel judéo-espagnol, acquis à Thessalonique. Elle est l'auteur de poèmes en cette langue de ses ancêtres sur la vie et la déportation des juifs de Thessalonique (19411943), préservant ce glorieux et douloureux héritage thessalonicien ${ }^{13}$.

\section{Les Thessaloniciens, les « voisins » des juifs}

Quelle fut l'attitude des «voisins » ${ }^{14}$ des juifs thessaloniciens? «Qui sont ceux qui, en 1945, ont pleuré leurs voisins disparus?».

À Thessalonique comme ailleurs, cacher des juifs était dangereux et difficile dans la ville où l'approvisionnement clandestin était plus aléatoire qu'à la campagne. Et pourtant des "voisins »y ont secouru des juifs, le site électronique de Yad Vashem ${ }^{15}$ affiche 58 noms de Justes parmi les Nations pour la ville de Thessalonique.

\footnotetext{
${ }^{13}$ Renée Levine Melammed, An ode to Salonika. The ladino verses of Bouena Sarfatty, Indiana University Press, Indiana Series in Sephardi and Mizrahi Studies, 2013. De la même auteure, «Bouena Sarfatty : her Life and Works in Ladino », dans David Bunis (ed.), Languages and literatures of Sephardic and Oriental Jews, Jérusalem, Bialik Institute, 2009, p. 120-127.

${ }^{14}$ Faut-il lire, dans ce terme, une allusion au livre de l'historien américain Jan Tomasz Gross d'origine polonaise : Les Voisins, Un massacre en Pologne 10 juillet 1941, traduit de l'américain par Pierre-Emmanuel Dauzat, Paris, Fayard, 2002 ? L'auteur y décrit le pogrom dont furent victimes les juifs de Jedwabne, en Pologne, perpétré par leurs voisins non juifs. L'analyse de ce pogrom par Gross a suscité des réactions en Pologne. Lors de journées d'études à l'université de Lille 3, en mars 2010 sur « Mille ans de Juifs en Pologne », l'historienne polonaise avait lucidement exposé ce débat, Edyta Gawron : «L'histoire des Voisins de J.T. Gross comme exemple d'incitation à un débat historique national », Tsafon $\mathrm{n}^{\circ}$ 60, automne 2010 - hiver 2011, p. 51-68. Depuis, la polémique a repris en Pologne où des nationalistes ont accusé JT Gross de déformer les faits par hostilité envers la Pologne. Ces accusations ont été contredites par quelques historiens polonais mais surtout par l'ensemble des historiens français et d'autres pays. La presse en a rendu compte. JT Gross est l'enfant d'un couple mixte (père juif et mère catholique) engagé dans la résistance polonaise. Lui-même avait participé à des démonstrations anticommunistes, en 1968, ce qui l'obligea à s'exiler aux USA.

${ }^{15}$ Consulté le 22 avril 2018.
} 
Et pourtant encore, le tableau au lendemain de la guerre est sombre pour la population juive, non seulement elle a été décimée par la déportation mais elle a été spoliée par ses «voisins». À commencer par la municipalité qui décida de détruire l'immense cimetière juif qui s'étendait sur $324000 \mathrm{~m}^{2}$ et abritait quelque 300000 tombes dont certaines très anciennes ${ }^{16}$. Des « voisins » ont pillé les maisons des juifs après leur départ, sans même l'ombre d'une compassion puisqu'une "page de l'Ancien Testament de [la] famille » de Bouena avait servi d'emballage à «ses pois chiches préférés, qu'elle avait achetés au vendeur arménien », une page d'un livre sacré et familial avait été souillée ! Le voisinage avait-il été si amical et sincère qu'il y paraissait avant la guerre puisque Bouena découvrit encore « le sac argenté, relique de la famille, [...] au bras d'une amie de famille chrétienne » et puisqu'une « autre Juive rescapée voit avec surprise, dans la maison d'une famille chrétienne amie » un «tapis familial»? Yiannis Boutaris se montre très sévère envers les profiteurs et s'il reconnaît que d'aucun " avait aidé [...] avait protégé », il dénonce avec force ceux qui « se sont cachés derrière leur petit doigt » : «...D’autres, nombreux, détruisaient, brûlaient, volaient, prenaient possession de l'espace et des biens des nombreux absents et des rares présents ».

Plus grave, sans doute, est l'exploitation des pierres tombales juives laissées à l'abandon après la destruction. Celles-ci ont été utilisées sans ménagement pour la reconstruction de la ville, après la guerre, par les Thessaloniciens. Si bien qu'un «passé silencieux, invisible mais présent » « poursuit » et « hante » les habitants jusqu'à aujourd'hui. Les pierres tombales parlent quand même de ce passé et le rendent visible : dans la «cour de l'église Saint-Dimitri», la principale église de Thessalonique, à l'hôpital associé à l'université Aristote «qui ont été construits sur une des nécropoles les plus importantes d'Europe » et ceci bien après la guerre. Ces dalles se touvent partout dans la ville : « autour du théâtre royal », sur «des routes et des trottoirs », devant la Tour blanche, fameux édifice de la ville etc.

Pour réparer «l'indifférence », il reste le souvenir des martyrs et comment exprimer ce souvenir? «Est-ce que nous les honorons en nous souvenant d'eux comme tels? Sommes-nous honorés de nous souvenir d'eux de cette façon?» Mais le souvenir fut lent à se manifester: «Quels monuments ont été érigés ? Quelles cérémonies ont eu lieu ?» questionne Yiannis Boutaris avec insistance. «Seule, la communauté

${ }^{16}$ Christos Zafiris, op. cit., p. 44. 
torturée et rackettée s'est battue pour se reconstruire et pour pleurer ses morts » ajoute-t-il pour mieux dénoncer l'oubli. La commémoration fut timide d'abord en 1962 avec la construction d'un «monument en mémoire des victimes » soit 20 ans après les événements insiste encore le maire de la ville, et non pas sur la place publique mais quasiment en un lieu privé : «Dans l'enceinte du nouveau cimetière juif ». Ce n'est qu'en 1997 qu'un « ...vrai monument a été érigé sur la place publique, il a été placé à la périphérie du centre-ville, dans un lieu peu visible». Plus de cinquante ans après la déportation des juifs, Thessalonique se souvenait mais sans ostentation. Puis, pour rendre plus visible la mémoire «...ce monument a été déplacé dans son environnement naturel, sur la place de la Liberté, cela a suscité plus de surprise que de satisfaction ». Il se situe désormais en un endroit bien en vue, face au port. En outre, tardivement encore, en 2014, une stèle a été « ...érigée dans l'enceinte de l'université Aristote afin de rappeler la destruction du cimetière $»^{17}$. Le souvenir est donc présent et interpelle le passant aux deux endroits emblématiques de la destruction de la communauté juive de Thessalonique : la place de la Liberté où se déroula le shabbat noir et le cimetière qui fut l'histoire de cette communauté. Il faudrait ajouter la plaque apposée sur le mur de la gare d'où partirent les convois de la déportation. Il faut encore ajouter la marche instituée, en 2013, par le maire Yiannis Boutaris, un dimanche proche de la date de départ du premier convoi du 15 mars 1943, de la place de la Liberté à la gare en commémoration, suivie par des Thessaloniciens non juifs et juifs descendants de déportés rescapés.

\section{Construire un avenir adossé au passé}

Et ce passé de Thessalonique est lointain, 2500 ans depuis l'Antiquité : «La municipalité de Thessalonique a de plus en plus conscience du poids de l'histoire que la ville doit soulever ». Toutefois, c'est l'histoire plus proche de ses concitoyens, celle du $\mathrm{XX}^{\mathrm{e}}$ siècle, que le maire entend sortir du « silence ». Cette conscience s'est particulièrement éveillée sous le mandat de Yiannis Boutaris (monuments, marches, discours officiels).

C'est une histoire aux «souvenirs difficiles, traumatisants » mais en plaçant les mots justes sur cette histoire qui a divisé, séparé les

\footnotetext{
${ }^{17}$ À cette occasion, le maire de Thessalonique prononça un discours qui dénonçait déjà la collusion entre l'occupant et la municipalité pendant la guerre; Pierre Sintès et Olivier Givre, op. cit., p. 214.
} 
Thessaloniciens, le maire entend revenir à une cohabitation «en harmonie », " promouvoir une culture de cohabitation et de respect mutuel afin que le lourd héritage du passé se transforme en tremplin pour un futur meilleur ». Et c'est bien sûr autour de cette place de la Liberté, lieu de tant d'événements fédérateurs ou diviseurs, que Thessalonique vivra «son passé, son présent, son futur ». Comme par le passé, le futur doit être multiculturel ou ne sera pas viable ${ }^{18}$. Car en dehors de la guerre civile de 1948 à laquelle participèrent des juifs : « Cinq jeunes étaient des héros juifs » et tombèrent lors de cette cette guerre, Yiannis Boutaris évoque les événements que la place de la Liberté a vécus. Thessalonique doit redevenir cette « mosaïque colorée ».

À partir de la place de la Liberté, Yiannis Boutaris peut dérouler l'histoire de sa ville, au $\mathrm{XX}^{\mathrm{e}}$ siècle. En 1908 d'abord, «...tous les Thessaloniciens musulmans, chrétiens et juifs ont fêté ensemble, la proclamation de la constitution ottomane » : après la rébellion des Jeunes Turcs, hostiles à l'autocratie exercée par Abdülhamid II et souvent retranchés en Macédoine plus éloignée de Constantinople, le sultan fut obligé d'accepter une constitution libérale, le 24 juillet 1908. Les partisans des Jeunes Turcs pouvaient compter parmi eux quelques Thessaloniciens juifs, tel Emmanuel Carasso (1862-1934) ${ }^{19}$.

Lieu donc de rassemblement démocratique, quinze années plus tard la place de la Liberté fut le lieu, cette fois, "de déracinement et d'exil » de la population thessalonicienne. Après une guerre d'indépendance qui s'étira de 1918 à 1923, la Turquie, dirigée désormais par Atatürk, obtint, par le traité de Lausanne (24 juillet 1923), la reconnaissance de ses nouvelles frontières et le déplacement de populations pour assurer une homogénéité religieuse dans le nouvel État, déplacement qui souvent, comme à Thessalonique, commença avant même la signature du traité. Ainsi les musulmans de Grèce durent-ils s'installer en Turquie alors que les chrétiens de Turquie devaient rejoindre la Grèce. La place de la Liberté fut donc le théâtre de ces transferts imposés : ce fut « le lieu d'où sont partis en 1922-1923 les anciens Thessaloniciens musulmans et où ont débarqué les nouveaux réfugiés d'Asie Mineure et du Pont-Euxin » ${ }^{20}$.

\footnotetext{
${ }^{18}$ Jusqu'à inclure l'islam dans ce futur multiculturel puisqu'il fit aussi partie du passé. Ibid., p. 219.

19 En 1909, Abdülhamid II fut interné à la villa de la famille juive Allatini, de Thessalonique.

${ }^{20}$ Sur l'histoire de la Turquie on peut se référer à Yves Ternon, Empire ottoman, le déclin, la chute, l'effacement, Paris, éditions du Félin/éditions Michel de Maule, 2002.
} 
Enfin après avoir été un lieu de rassemblement et un lieu de déracinement, la place de la Liberté fut: «un lieu de martyre, d'humiliation publique des Juifs thessaloniciens, où le samedi noir du 11 juillet 1942, les Allemands ont diffamé 9000 Juifs devant les chrétiens grecs $»$.

C'est ce dernier événement douloureux que le maire ne veut pas effacer de la mémoire des habitants de sa ville. « À quelques mètres de là [de la place de la Liberté], le musée de l'Holocauste symbolisera notre honte pour tout ce qui s'est passé $[\ldots]$ pendant et après la guerre $[\ldots]$ C'est une dette de la ville envers ses Juifs, en tant que Thessaloniciens, Grecs et Sépharades». Mais dans son «pari personnel», Yiannis Boutaris ne limite pas la dette aux seuls Thessaloniciens car : «Le musée dépasse la ville et la Grèce et replace Thessalonique comme métropole des juifs sépharades de Méditerranée ». Il faut redonner sa juste place, dans la mémoire internationale, à «l'Holocauste» qui anéantit les communautés juives des Balkans, trop souvent occulté par la mémoire de l'extermination des juifs d'Europe centrale et orientale. L'ambition, justifiée, est donc de créer un musée de «l'Holocauste» pour toutes les communautés, dont Yiannis Boutaris dresse une liste non exhaustive, un musée «d'envergure internationale», parallèlement au musée de Washington ou au Mémorial de la Shoah à Paris ou encore au musée de Berlin voire le récent musée en Pologne. Thessalonique redeviendra alors, incontestablement, «le lieu de mémoire» des judaïcités balkaniques, comme elle fut la « Jérusalem des Balkans ».

Au-delà du souvenir ou de la mémoire, Yiannis Boutaris nourrit l'ambition d'instaurer une œuvre pédagogique. Le musée sera : «...Un lieu où les habitants de la terre entière, en particulier les jeunes, apprendront les conséquences du bafouement des droits de l'homme ». Il signe ainsi les pages d'un avenir apaisé et harmonieux.

Par ce discours, le maire de Thessalonique réconforte la petite communauté juive de Thessalonique. Il confirme ses engagements pour entretenir la mémoire de la Shoah, non seulement à Thessalonique mais aussi dans les Balkans. Sans doute bouscule-t-il le confort de ses administrés en évoquant ce passé douloureux, comme il l'a fait sur bien d'autres sujets. Sa démarche est d'autant plus courageuse qu'elle se place dans le contexte de crise économique que connaît la Grèce actuellement et le contexte de difficultés politiques avec l'émergence du parti Aube dorée, qui se situe à l'extrême droite de l'échiquier politique de la Grèce 
et qui a fait son entrée au parlement d'Athènes en 2012. Le parti est ouvertement antisémite et remet en cause la réalité de la Shoah. Certes cette idéologie et ce courant restent minoritaires mais ils progressent lentement. Les initiatives de Yiannis Boutaris, maire de Thessalonique, peuvent contrer ces dérives.

Les monuments mémoriels de la Shoah à Thessalonique

(Photos : Danielle Delmaire, mars 2018)

Place de la Liberté

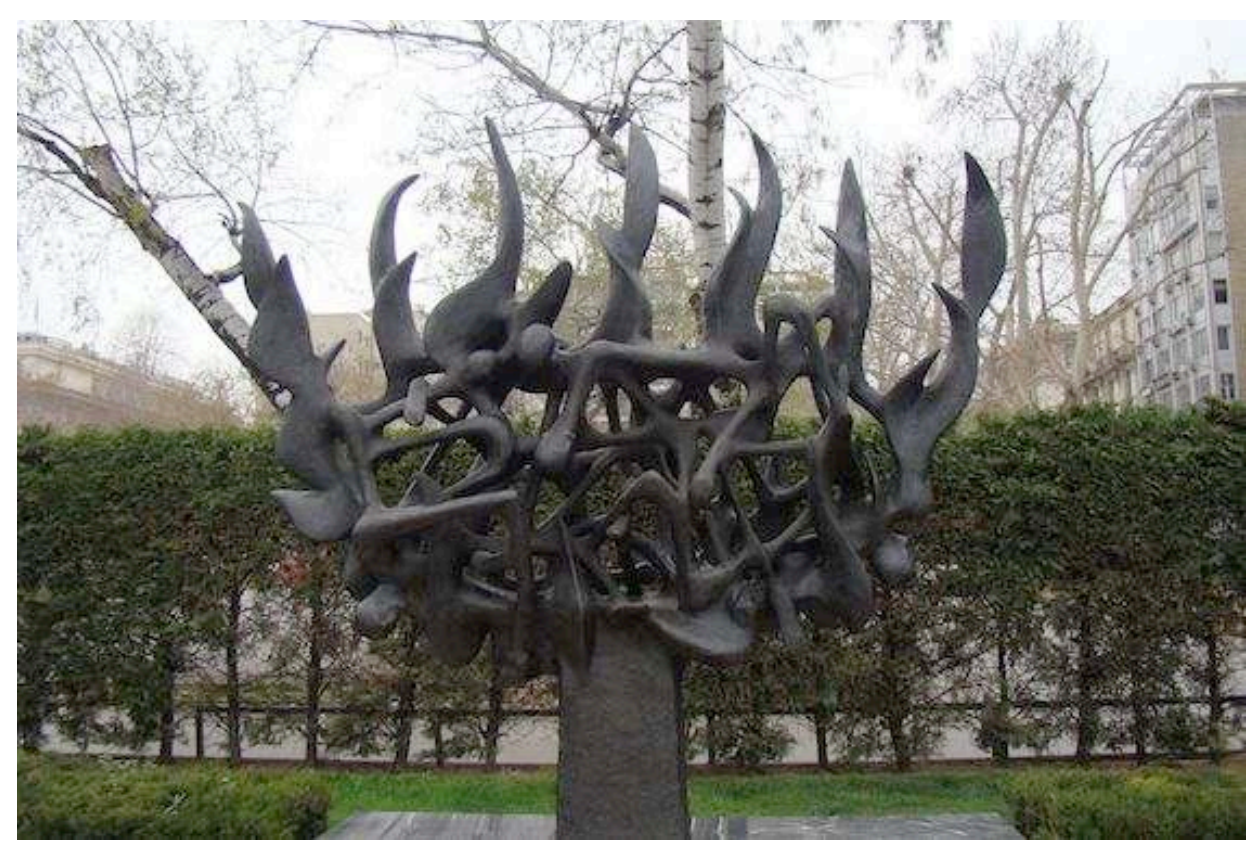

Le monument représente sept flammes

qui s'élèvent vers le ciel et évoquent la ménorah.

Dans ces flammes s'entremêlent des corps humains décharnés et amoncelés les uns sur les autres. 
Les monuments mémoriels de la Shoah à Thessalonique

Monument à l'université Aristote

Inauguré en 2014

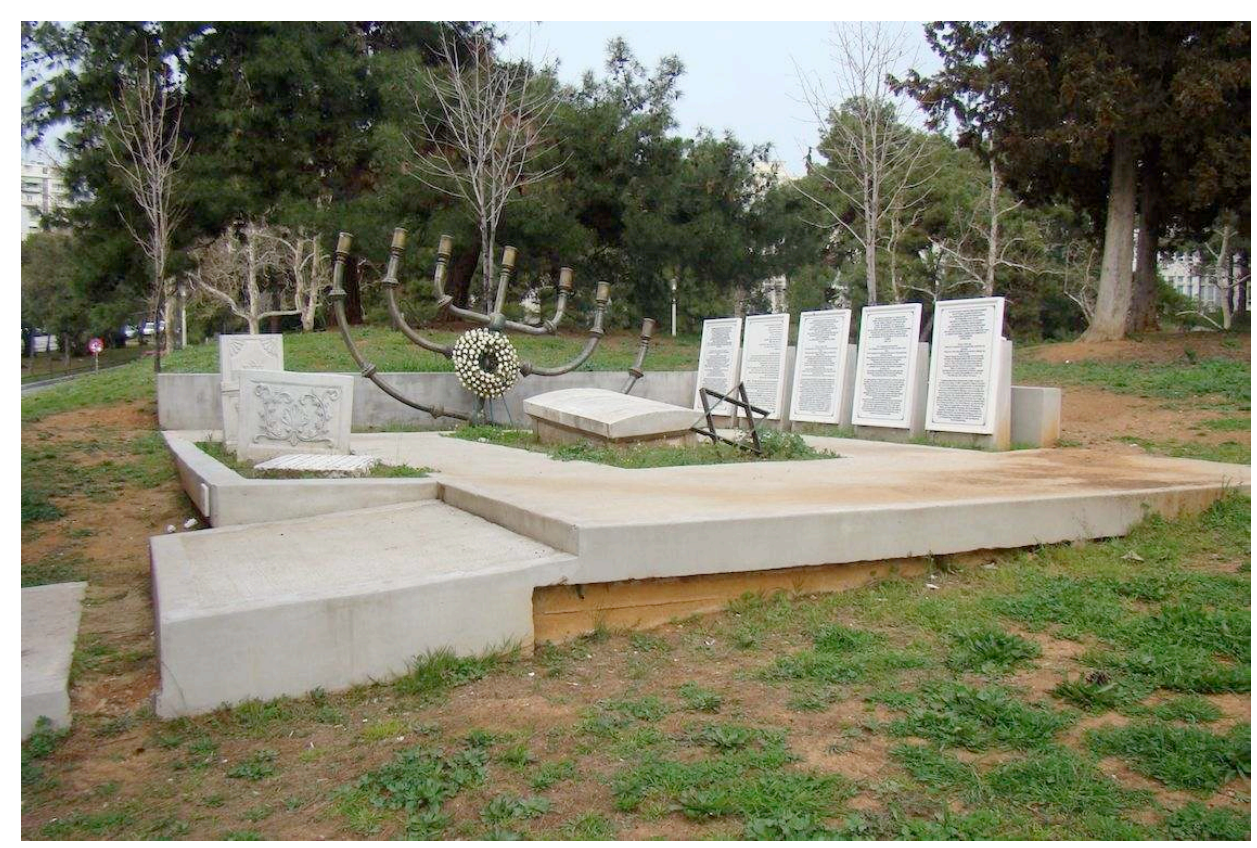

Une ménorah et une étoile de David basculées entourent une pierre tombale.

À gauche, des fragments de pierres tombales rappellent ce que fut originellement l'emplacement de l'université : le cimetière juif.

À droite, des plaques évoquent, en plusieurs langues, le sort des juifs de Thessalonique durant la Seconde Guerre mondiale. 
Les monuments mémoriels de la Shoah à Thessalonique

Plaque sur la façade de la gare

\section{DEDICATED TO THE SACRED MEMORY OF THE 50000 GREEK JEWS OF THESSALONIKI WHO. FROM MARCH UNTIL AUGUST 1943, WERE TAKEN BY THE NAZI CONQUERORS TO THE OLD RAILWAY STATION, PILED UP INTO CLOSED LIVESTOCK WAGONS AND WERE DEPORTED TO THE CAMPS OF AUSCHWITZ - BIRKENAU WHERE THEY MET A MARTYR'S DEATH}

La plaque est apposée sur la façade de l'ancienne gare de Thessalonique.

C'est à cette gare qu'aboutit la marche du souvenir qui a lieu, chaque année, un dimanche proche du 15 mars, jour du premier convoi parti vers Auschwitz, en 1943.

Le départ de la marche est fixé place de la Liberté. 